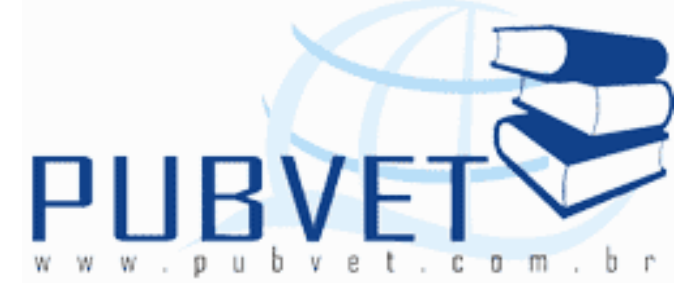

PUBVET, Publicações em Medicina Veterinária e Zootecnia.

\title{
Levantamento de cães vacinados contra a Leishmaniose visceral no município de Mossoró-RN
}

Andréa Cristina Capriata Silva ${ }^{1}$, Josué de Oliveira Moreira ${ }^{2}$, Shirlei Barros de Medeiros $^{3}$

${ }^{1}$ Doutoranda em Parasitologia UFPEL, Pelotas-RS - Bolsista FAPERGS/CAPES; Email: deiavet@hotmail.com

${ }^{2}$ Docente IFRN, Mossoró-RN; Email: moreira7@hotmail.com

${ }^{3}$ Acadêmica do curso de Medicina Veterinária UFERSA, Mossoró-RN; Email: cammylle.torlone@hotmail.com

\section{Resumo}

O uso de vacina licenciada contra leishmaniose visceral canina (LVC) está difundido em todo Brasil como ponto importante da profilaxia da doença em grandes centros urbanos. O objetivo do trabalho foi realizar um levantamento epidemiológico dos cães vacinados contra a LVC no município de Mossoró-RN nos anos de 2009 e 2010. Participaram do estudo quatro clínicas veterinárias ( $57 \%)$, os dados obtidos foram catalogados em fichas padronizadas para cada ano específico e individualizada. As fichas continham informações que buscavam indagar questões sobre: o ano de vacinação, a idade do animal, a raça, o sexo e o bairro que o animal residia. Em seguida foram agrupados em planilhas na plataforma Microsoft Excel e posteriormente analisados. No ano de 2009 e 2010 foram vacinados 214 e 228 animais respectivamente, totalizando 
SILVA, A.C.C., MOREIRA, J.O., MEDEIROS, S.B. Levantamento de cães vacinados contra a Leishmaniose visceral no município de Mossoró-RN. PUBVET, Londrina, V. 6, N. 26, Ed. 213, Art. 1418, 2012.

442 animais da espécie canina. As fêmeas e os animais adultos apresentaram como maioria nesse estudo $226(51 \%)$ e 277 (67\%) respectivamente. A raça poodle apresentou como maior número de vacinados, $131(29,6 \%)$ animais. $O$ bairro Nova Betânia destacou-se com o maior número de vacinados, sendo 165 $(37,3 \%)$ cães imunizados nos dois anos. O número de animais vacinados pode ser considerado pequeno se comparado com a população canina existente no município. Mesmo assim, mostra-se uma forte tendência de crescimento de cães imunizados contra LVC à medida que a mesma se torna mais popular e acessível. Mostrando-se dessa forma um caminho a ser seguido no controle dessa importante zoonose nos centros urbanos.

Palavras-Chave: Leishmaniose Visceral, cães imunizados, zoonose

\title{
Search dogs vaccinated against visceral Leishmaniasis in the city of Mossoró-RN
}

\begin{abstract}
The use of licensed vaccine against canine visceral leishmaniasis (CVL) is widespread throughout Brazil as an important issue of prevention of disease in large urban centers. The objective of this study was realized an epidemiological survey of dogs immunized against Leishmaniose Visceral in Mossoró-RN, during the years 2009 and 2010. The study included four veterinary clinics $(57 \%)$, the data were categorized into standardized forms for each specific year and individualized. The records contained information about: the year of vaccination, the animal's age, race, sex and neighborhood where the animal lived. The data was grouped into spreadsheets on Microsoft Excel and then analyzed. In 2009 and 2010 were vaccinated 214 animals and 228 respectively, totaling 442 animals of the canine species. Females and animals adults were showed as the most number in this study, with $226(51 \%)$ and $277(67 \%)$ respectively. The Poodle breed had a greater number of vaccinees, 131 (28\%) animals. The neighborhood of Nova Betânia stood out with the highest number of vaccinated, and 168 (37\%) dogs were immunized. The
\end{abstract}


SILVA, A.C.C., MOREIRA, J.O., MEDEIROS, S.B. Levantamento de cães vacinados contra a Leishmaniose visceral no município de Mossoró-RN. PUBVET, Londrina, V. 6, N. 26, Ed. 213, Art. 1418, 2012.

number of vaccinated animals can be considered small to compared with the existing canine population in the city of Mossoró, RN. However, It shows a new way forward in controlling this important zoonotic disease in urban centers.

Keywords: Visceral Leishmaniasis, dogs immunized, zoonosis

\section{INTRODUÇÃO}

A Leishmaniose Visceral (LV) é uma das mais importantes zoonoses emergentes de origem parasitária, sendo considerada a segunda maior enfermidade no mundo causada por protozoários, perdendo apenas para a Malária. Esta doença tem distribuição mundial, sendo causada por protozoários do gênero Leishmania. No Novo Mundo é causada pelo protozoário Leishmania (Leishmania) chagasi, enquanto que Leishmania (Leishmania) infantum é descrita em alguns países da Ásia e do Mediterrâneo (WHO, 2008).

No Brasil, as estratégias de controle da doença estão baseadas no diagnóstico e tratamento precoce de casos humanos, no controle dos vetores por meio do uso de inseticidas e na deteç̧ão dos cães infectados por análises parasitológicas e sorológicas (Reação de Imunofluorescência Indireta - RIFI e ensaio imunoenzimático - ELISA) com posterior eutanásia dos animais positivos (BRASIL, 2003).

No estado do Rio Grande do Norte a LV passou a representar um importante problema de saúde pública a partir de 1989 e a comparação entre coeficientes de prevalência da doença nos estados do Nordeste mostra que o estado do Rio Grande do Norte, nos últimos dez anos, foi superado apenas pelos estados de Piauí e Sergipe (XIMENES et al., 1999). Durante um inquérito canino realizado pela Secretaria Municipal de Saúde de Mossoró-RN, entre 2001 e 2002, dentre 16.686 amostras de sangue canino examinadas, foi registrada prevalência em torno de 3,8\% (TIERZO, 2003).

Uma forma de prevenção para que os cães não adquiram essa doença é a vacinação dos animais sadios. Após o registro no Ministério da Agricultura, em 11 de junho de 2003 (MENZ, 2006). Os autores concluíram que a vacina 
SILVA, A.C.C., MOREIRA, J.O., MEDEIROS, S.B. Levantamento de cães vacinados contra a Leishmaniose visceral no município de Mossoró-RN. PUBVET, Londrina, V. 6, N. 26, Ed. 213, Art. 1418, 2012.

induz significante, duradouro e forte efeito protetor contra LVC no campo (BORJA-CABREIRA et al., 2002). É considerado um ponto importante da profilaxia da doença as vacinas contra LV canina atualmente em comercialização. Entretanto, para renovação de registro, seus fabricantes tiveram que executar os estudos da fase III para análise junto ao Ministério da Agricultura (MAPA) e Ministério da Saúde (MS). No momento, aguarda-se a liberação dos resultados finais (Carta de Brasília, 2010). O laboratório responsável pela vacina Leishmune $\mathbb{R}$ indica a imunização de animais totalmente sadios a partir do quatro meses de idade, com a comprovação da realização de exames laboratoriais que comprovem serem soronegativos. Para que os animais sejam considerados imunes a doença é necessária a aplicação por via subcutânea de três doses consecutivas nos animais com intervalos de 21 dias e reforços anuais, de acordo com a data da primeira administração. (Nogueira, et al. 2005)

Resultados associados ao uso da vacina Leishmune $\AA$ em relação a sua eficácia, segurança e imunogenicidade demonstraram que o produto confere forte soroconversão após completa vacinação e significativo efeito protetor nos cães vacinados em áreas endêmicas, sendo estes resultados semelhantes aos obtidos nos trabalhos que antecederam a aprovação do produto comercial (Borja-cabrera, et al. 2002 e 2008; Silva, et al. 2001).

Diante disso, objetivou-se realizar o levantamento epidemiológico dos cães imunizados contra a Leishmaniose Visceral Canina no município de Mossoró-RN nos anos de 2009 e 2010.

\section{MATERIAL E MÉTODOS}

O estudo foi desenvolvido na cidade de Mossoró-RN, durante os anos de 2009 e 2010. O município possui um total de sete clínicas veterinárias legalizadas junto ao Conselho Regional de Medicina Veterinária do Rio Grande do Norte-CRMV-RN. Todas as clínicas foram convidadas a participarem do estudo, porém apenas quatro clínicas se propuseram a colaborar com o levantamento epidemiológico de cães vacinados contra LVC. 
SILVA, A.C.C., MOREIRA, J.O., MEDEIROS, S.B. Levantamento de cães vacinados contra a Leishmaniose visceral no município de Mossoró-RN. PUBVET, Londrina, V. 6, N. 26, Ed. 213, Art. 1418, 2012.

Os dados obtidos foram catalogados em fichas padronizadas para cada ano específico e individualizada para cada clínica avaliada. As fichas continham informações que buscavam indagar questões sobre: o ano de vacinação, a idade do animal, a raça, o sexo e o bairro que o animal residia. Os dados obtidos foram agrupados e analisados em planilhas na plataforma Microsoft Excel.

\section{RESULTADOS E DISCUSSÃO}

No ano de 2009 e 2010 foram vacinados 214 e 228 cães respectivamente, totalizando 442 animais da espécie canina. Esses números revelam que houve um pequeno crescimento na demanda da vacinação na cidade de Mossoró-RN, o que deve estar relacionado a acessibilidade das informações dos proprietários sobre os riscos causados pela doença e um grande número de animais sacrificados por serem portadores da doença.

As fêmeas vacinadas mostrou-se percentual acima dos machos, com um total de 226 (51\%) animais imunizados em relação aos machos que apresentou 216 (49\%) cães vacinados contra a doença.

A grande maioria, ou seja, $41 \%$ dos cães vacinados apresentavam idade entre 1 ano a 3 anos, em segundo lugar os cães com idade acima de 3 anos com 28,7\%. Os cães entre 6 meses a 1 ano de idade apresentaram um percentual de $23,3 \%$ em relação a todos animais avaliados. Os filhotes abaixo de 6 meses possuíram apenas 7\% do total de cães vacinados (Figura 1). Nesse estudo foi observado que os animais adultos foram considerados os mais imunizados contra a doença em relação aos mais jovens. Isso demonstra a necessidade de informar e sensibilizar os proprietários para a vacinação dos cães na idade recomendada. Isso demonstra o risco que os cães estão expostos após os quatro meses, idade recomendada para o início da imunização anti-LVC. 
SILVA, A.C.C., MOREIRA, J.O., MEDEIROS, S.B. Levantamento de cães vacinados contra a Leishmaniose visceral no município de Mossoró-RN. PUBVET, Londrina, V. 6, N. 26, Ed. 213, Art. 1418, 2012.

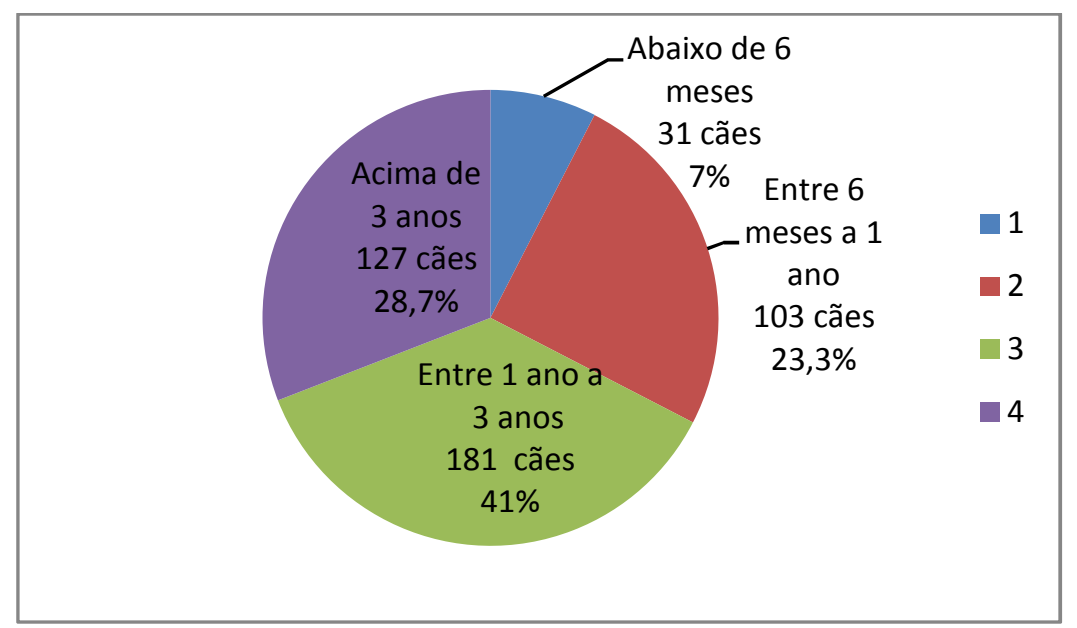

Figura 1: Faixa etária e percentual de animais vacinados contra LVC na cidade de Mossoró-RN, 2011.

De acordo com a Tabela 1 podemos observar que a maioria dos animais vacinados era da raça Poodle e em segundo lugar temos os animais Sem Padrão de Raça Definida (SRD). Observou-se também a variedade de raças existentes no município demonstrando uma diversidade de gostos e escolhas.

Há uma variedade maior de raças de cães de companhia que foram submetidos à imunização quando comparados com os cães de guarda (Tabela 1), mostrando que os proprietários de cães de companhia têm maior valor afetivo, sensibilidade e consciência com seus animais, o que resulta em animais com maiores expectativas de vida.

Já os cães de guarda permanecem no peridomicílio na maior parte de sua vida e são em sua maioria os animais soropositivos nas áreas urbanas. Esses valores retratam a importância da conscientização dos proprietários dos cães de guarda para que imunizem seus cães por estarem mais susceptíveis a contraírem a doença em decorrência da sua maior permanecia a céu aberto, expostos a facilidades de acesso as picadas de flebotomíneos. 
SILVA, A.C.C., MOREIRA, J.O., MEDEIROS, S.B. Levantamento de cães vacinados contra a Leishmaniose visceral no município de Mossoró-RN. PUBVET, Londrina, V. 6, N. 26, Ed. 213, Art. 1418, 2012.

Tabela 1. Principais raças e percentuais de cães vacinados contra Leishmaniose Visceral no município de Mossoró-RN, 2011.

\begin{tabular}{|l|c|}
\hline \multicolumn{1}{|c|}{ Raça } & Animais vacinados \% \\
\hline Poodle & $131(29,6 \%)$ \\
\hline SRD & $114(25,8 \%)$ \\
\hline Pinscher & $39(8,8 \%)$ \\
\hline Pit Bull & $31(7 \%)$ \\
\hline Yorkshire & $29(6,6 \%)$ \\
\hline Dalmata & $26(5,9 \%)$ \\
\hline Golden Retriever & $20(4,5 \%)$ \\
\hline Rottweiler & $16(3,6 \%)$ \\
\hline Shitzu & $10(2,3 \%)$ \\
\hline Labrador & $9(2 \%)$ \\
\hline Outras raças & $17(3,9 \%)$ \\
\hline Total & 442 \\
\hline
\end{tabular}

O bairro com maior número e percentual de cães vacinados foi Nova Betânia, esse é considerado nobre por ter proprietários de cães com alto poder aquisitivo da cidade de Mossoró. A vacina contra Leishmaniose Visceral Canina ( $L V C)$, ainda é considerada de alto custo para grande parte da população brasileira, o que inviabiliza que pessoas com menor poder aquisitivo possam estar adquirindo e consequentemente proteger os seus animais de estimação.

Foi realizado inquérito canino para LV em 1.401 cães no bairro Santo Antônio em 2009 e observou-se uma alta prevalência de 24\% (333 animais positivos) e 118 ( $8 \%$ ) cães com reações indeterminadas. Foram capturados para sacrifício $150(49,9 \%)$ animais, os demais teve as seguintes justificativas para a não entrega de seus animais: morte do animal, mudança de bairro, viagem para outro município, recusa, repetição de um novo exame particular, ausência do proprietário em casa para fazer a entrega do cão e a dificuldade 
SILVA, A.C.C., MOREIRA, J.O., MEDEIROS, S.B. Levantamento de cães vacinados contra a Leishmaniose visceral no município de Mossoró-RN. PUBVET, Londrina, V. 6, N. 26, Ed. 213, Art. 1418, 2012.

de recursos humanos e a indisponibilidade logística para manter a carrocinha a disposição desse serviço nesse bairro frente a diversos pontos de captura de cães no município. No mesmo estudo, observou-se que nesse mesmo bairro no período de 2006 a 2009 foram confirmadas 12 casos LV humana (Oliveira Filho, 2009). Diante dessa fotografia, que demonstrou a complexidade da LVC e LVH do bairro Santo Antônio somando-se a isso o baixo percentual de cães vacinados $(1 \%)$, leva-nos a repensar e reavaliar o Programa Nacional de Controle da LV canina e humana no município de Mossoró.

Tabela 2: Número de animais vacinados e percentual de acordo com o bairro da cidade de Mossoró-RN, 2011.

\begin{tabular}{|l|c|}
\hline \multicolumn{1}{|c|}{ Bairro } & Animais vacinados \\
\hline Nova Betânia & $165(37,3 \%)$ \\
\hline Boa Vista & $72(16,3 \%)$ \\
\hline Alto de São Manuel & $67(15,2 \%)$ \\
\hline 12 Anos & $40(9,1 \%)$ \\
\hline 30 de Setembro & $30(6,79 \%)$ \\
\hline Abolição & $28(6,3 \%)$ \\
\hline Centro & $13(2,9 \%)$ \\
\hline Liberdade & $5(1,1 \%)$ \\
\hline Alto da conceição & $4(0,9 \%)$ \\
\hline Costa e Silva & $4(0,9 \%)$ \\
\hline Santo Antônio & $3(0,7 \%)$ \\
\hline Outros & $11(2,5 \%)$ \\
\hline Total & 442 \\
\hline
\end{tabular}

Segundo Palatnik-de-Sousa, C.B. et al. 2009, observaram a redução dos casos de LVC e LVH após vacinação de cães com Leishmune ${ }^{\circledR}$ em áreas endêmicas do Brasil, em Araçatuba após vacinação foi observado uma redução de $25 \%$ da LVC com um declínio de $61 \%$ de casos humanos, indicando o efeito 
SILVA, A.C.C., MOREIRA, J.O., MEDEIROS, S.B. Levantamento de cães vacinados contra a Leishmaniose visceral no município de Mossoró-RN. PUBVET, Londrina, V. 6, N. 26, Ed. 213, Art. 1418, 2012.

aditivo da vacinação com Leishmune $\Re$ de $5,7 \%$ dos cães sãos (1.419 cães), sobre o sacrifício regular de cães.

Deve existir uma maior preocupação das autoridades sanitárias em realizar campanhas de priorização dos bairros com maior complexidade epidemiológica negativa. O controle profilático do uso da vacina anti LVC em massa parece ser uma alternativa que deve ser testada e avaliada na perspectiva de encontrar caminhos eficazes contra essa terrível zoonose que está muito bem adaptada ao meio urbano.

Segundo Palatnik-de-Sousa, C.B. et al. 2009, concluíram que a redução das incidências canina e humana está diretamente correlacionada com o aumento do número de cães vacinados, em conformidade com o efeito aditivo de controle da vacinação sobre o sacrifício de cães, reduzindo o reservatório do parasita, protegendo os cães e, dessa forma , reduzindo o risco de transmissão da leishmaniose para humanos, tornando-se uma nova e eficaz ferramenta de controle.

O número de animais vacinados parece ser pequeno se comparado com a população canina existente na cidade de Mossoró, porém pode-se vislumbrar um caminho seguro e menos traumático para se evitar a mortandade de cães eutanasiados por essa enfermidade pelos Centros de Controle de Zoonoses. A não disponibilidade de informações sobre o número de animais vacinados para LVC pelas demais clínicas e profissionais médicos veterinários autônomos dificultaram uma análise mais precisa do número de cães vacinados.

A vacinação de cães anti-LVC parece se mostrar um novo caminho profilático seguro a ser seguido no controle dessa importante zoonose nos grandes centros urbanos, mesmo diante do aguardo da liberação dos resultados finais das avaliações dos protocolos da fase III das vacinas contra LV canina. 
SILVA, A.C.C., MOREIRA, J.O., MEDEIROS, S.B. Levantamento de cães vacinados contra a Leishmaniose visceral no município de Mossoró-RN. PUBVET, Londrina, V. 6, N. 26, Ed. 213, Art. 1418, 2012.

\section{CONCLUSÃo}

A maioria dos cães vacinados foi do sexo feminino, considerados adultos e serviam de companhia, esses animais possuem melhores condições de vida proporcionando maior longevidade.

O bairro mais nobre da cidade foi o que possuiu a maior quantidade de cães imunizados contra LVC, mostrando que a vacina ainda possui um custo elevado, o que seleciona os consumidores e dificulta a diminuição de cães eutanasiados na cidade de Mossoró.

\section{AGRADECIMENTOS}

A FAPERGS/CAPES pela concessão de bolsa de estudo para realização da pesquisa.

\section{REFERÊNCIAS}

FILGUEIRA, K. D.; RÊGO, R. O.; ALVES, N. D. Detecção de anticorpos anti-Leishmania chagasi em cães da área rural do município de Mossoró, Rio Grande do Norte. Ciência Animal Brasileira, v. 5, p. 166-168, 2004.

OLIVEIRA FILHO, F.C. Distribuição espacial da Leishmaniose Visceral no bairro Santo Antônio em Mossoró-RN, Monografia de graduação do Curso de Medicina Veterinária, UFERSA, 2010.

BORJA-CABRERA, G.P.; MOREIRA,M.A.B.; NOGUEIRA, F.S.; SANTOS,F.N.; MENZ, I.; PARRA, L.E.; XU,Z.; CHU,H.J.; PALANTNIK-de-SOUSA,C.B.; LUVIZOTTO,M.C.R. Leishmune ${ }^{\circ}$ vaccine blocks the transmission of canine visceral leishmaniasis absence of Leishmania parasites in blood, skin and lymph nodes of vaccinated exposed dogs. Revista Vaccine. 2005, p. 1-6. Disponível em: < http://www.elsevier.com/locate/vaccine>.

BORJA-CABRERA, G.P. et al. Long lasting protection against kala-azar using the FML-QuilA saponin vaccine in an endemic area of Brazil. Vaccine 20 (2002) 4991 - 4997.

BORJA-CABRERA, G.P. et al. Immunogenicity assay of the Leishmune ${ }^{\circ}$ vaccine against canine visceral leishmaniasis in Brazil. Vaccine 26 (2008) 4991-4997.

SILVA, V.O. et al. A phase III Trial of efficacy of the FML-vaccine against canine kala-azar in an endemic area of Brazil (São Gonzalo do Amarante,RN). Vaccine 19 (2001) 1082-1092.

BRASIL. Ministério da Saúde. Secretaria de Vigilância em Saúde. Departamento de Vigilância Epidemiológica. Manual de Vigilância e Controle da Leishmaniose Visceral/ Ministério da Saúde, Secretaria de Vigilância em Saúde, Departamento de Vigilância Epidemiológica. - Brasília: Ministério da Saúde, 2003. 
LIMA, V. M. F.; PEIRO, J. R.; VASCONCELOS, R. O. IL-6 é produzida em cães naturalmente infectados com Leishmania chagasi. In: CONGRESSO BRASILEIRO DE PARASITOLOGIA VETERINÁRIA, 14., 2006, Ribeirão Preto. Anais... Ribeirão Preto, 2006. p. 322.

MENZ, I. Leishmune $尺$ - Desenvolvimento e Resultados Atuais (dez 2005). Anais do 10 Fórum sobre Leishmaniose Visceral Canina. Colégio Brasileiro de Parasitologia Veterinária. Faculdade de Ciências Agrárias e Veterinárias da Universidade Estadual Paulista. Jaboticabal(SP), 2006.

GONTIJO, C.M.F.; MELO, M.N. Leishmaniose visceral no Brasil: quadro atual, desafios e perspectivas. Revista Brasileira de Epidemiologia, São Paulo, v.7, n.3, p.338-349, 2004.

MOURA S.T. Diagnóstico de leishmaniose canina na área urbana do Município de Cuiabá, Estado de Mato Grosso, Brasil. Brazilian Journal of Veterinary Research and Animal Science, São Paulo, v.36, n.113, p. 101-102, 1999.

NOGUEIRA, F.S.; MOREIRA, M.A.; BORJA-CABREIRA, G.P.; SANTOS, F.N.; MENZ, I.; PARRA, L.E.; XU, Z.; PALANTIK-de-SOUSA, C.B.; LUVIZOTTO, M.C.R. Leishmune ${ }^{\circledR}$ vaccine blocks the transmission of canine visceral leishmaniasis. Absence of Leishmania parasites in blood, skin and lymphnodes of vaccinated exposised dogs. Revista Vaccine, mai 2005, p. 1-6. Disponível em: < http://www.elsevier.com/locate/vaccine>.

PALATNIK DE SOUZA, C. B.; PARAGUAI DE SOUZA, E.; GOMES, E. M.; BOROJEVIC, R. Experimental murine Leishmania donovani infection immunoprotection by the Fucose Mannose Ligand (FML). Brazilian Journal of Medical Biological Research, Ribeirão Preto, v. 27, p. 547-551, 1994.

PALATNIK DE SOUZA, C. B.; Silva-Antunes, I.; Morgado, A. A.; Menz, I.; Palatnik, M.; Lavor, C. Redução da incidência de leishmaniose visceral humana e canina após vacinação de cães

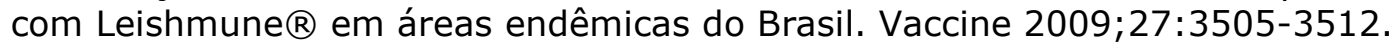

TIERZO, F. L. Estudo retrospectivo da Leishmaniose Visceral no município de Mossoró-RN, 2003. 24. Monografia. (Graduação em Medicina Veterinária) - Universidade Federal Rural do Semi-Árido (UFERSA).

WHO - Research to support the elimination of visceral leishmaniasis. Disponível em: http://apps.who.int/tdr/svc/publications/about-tdr/annual-reports/visceralleishmaniasiselimination-2008 . Acesso em: 11/jan./2012.

XIMENES, M. F.; CASTELLÓN, E. G.; OLIVEIRA, R. M. Leishmaniose visceral no Rio Grande do Norte: aspecto eco-epidemiológico. In: CONGRESSO BRASILEIRO DE PARASITOLOGIA, 1999, Poços de Caldas. Anais... Poços de Caldas, 1999. 